\title{
Improving author experience
}

\section{Nature Cell Biology explores avenues to optimize the peer-review process and improve author experience.}

In the past year, the Nature Cell Biology editorial team has concentrated its efforts on refining the peer review process on two fronts: (1) developing 'guided portable peer review', providing authors whose papers were peer reviewed but declined at Nature Cell Biology with an informed recommendation for submission at an alternative title in the Nature family and (2) renewing our efforts to provide strong editorial guidance to authors revising their submissions in response to referee recommendations.

At conferences and lab visits we consistently hear that a central failing of the current review and research assessment system is that academic success is contingent on publication in elite journals, and the path to publication at highly selective journals has become untenably arduous. Authors often endure multiple, multi-year cycles of review and revision. Rejected authors are then faced with the daunting prospect of starting afresh at a new journal. We hope the two improvements that we have developed will go some way towards mitigating these pressing concerns for our authors, and will enable a productive and rapid path to publication.

The guided portable peer review process represents an important step in this direction. For a select subset of manuscripts that have been reviewed but declined at Nature Cell Biology and that are, following extensive editorial discussions, determined to be strong candidates for eventual publication at Nature Communications (for example, if the manuscript is technically sound but does not represent a sufficient scientific advance for Nature Cell Biology), we consult with our colleagues at Nature Communications ahead of sending a decision, and provide detailed guidance to authors. Typically, this includes a commitment to re-review at Nature Communications and a prioritization of revisions that would need to be addressed for consideration there. The author response to this initiative has been enthusiastic. Over a three-month timespan where we tracked author uptake, $76 \%$ of the Nature Cell Biology authors who were provided with a guided recommendation transferred their reviewed manuscript to Nature Communications. All transferred manuscripts were eventually published in Nature Communications and the majority of authors felt that the transfer resulted in a more rapid path to publication than if they had started anew at a different journal. Having insight into potential future steps at a new journal and saving time and effort that would have been spent in a fresh review cycle elsewhere were the most important considerations that led authors to accept the recommendation and transfer to Nature Communications. Aside from the immediate and obvious value to our authors, portable peer review has important benefits for the community in reducing the overall effort expended by referees in assessing a manuscript time and again at multiple different journals.

More broadly, Nature Publishing Group (NPG) has also modified the process for facilitating manuscript transfer between journals so that, for select manuscripts that are perceived as having a high potential for success at a sister title, editors may consult confidentially with their colleagues at that title. Peer review consortia that facilitate sharing of referee reports between journals have existed for some time, most notably the Neuroscience Peer Review Consortium, which was formed in 2008. Within NPG, we too have long had a manuscript transfer service (http:// go.nature.com/PY7jLh) that allows authors to transfer manuscripts (reviewed or not) from one Nature journal to another, thus avoiding time and effort spent in reformatting, resubmitting and undergoing fresh cycles of review with new referees. If the manuscript has been reviewed, the referee reports and identities can be transferred to another Nature journal of the author's choosing. The receiving journal will then typically use the existing reports and referees to reach a decision on the transferred manuscripts, although in some cases it may be necessary to recruit additional experts, for example if revisions include specialist approaches that were absent in the original, reviewed submission. As described above, this consultation process has the potential to provide an informed recommendation to authors and streamline the review process. The ultimate decision of where to submit and how to proceed with the manuscript remains entirely within the author's control; authors may optout of such consultations at submission stage. While the modifications to the transfer process allow us to leverage our collective editorial strength in the service of our authors, editorial independence - a cornerstone of the editorial philosophy at NPG - remains unchanged with individual journals continuing to retain complete autonomy over editorial decisions.

Another area of ongoing dissatisfaction with existing peer review processes are the open-ended requests for revisions that are not focused on strengthening the core findings of a study. In the many conversations that Nature Cell Biology editors have had with researchers over the years, it has become clear that navigating the thicket of referee comments is a major hurdle for most researchers. This year, we have redoubled our efforts to map referee requests onto specific editorial recommendations and to distil for authors the key priorities that would need to be addressed in revision. Where appropriate, editors also provide unequivocal feedback on points that we view to be beyond the scope of the study and thus would not need to be addressed for further consideration at Nature Cell Biology. Feedback from authors to these renewed endeavours has been gratifyingly positive. Ancillary to these efforts, Nature Cell Biology has always been committed to providing an efficient, fair and constructive path to publication. This year, we are pleased to note that of the papers accepted at the journal from January-October 2014, 91.8\% underwent only one major round of revision, with a median duration from submission to acceptance (including the time spent revising) of approximately 7 months.

Editorial policies and procedures at Nature Cell Biology and Nature Publishing Group as a whole have typically evolved to meet the emerging needs of the research process, the authors, the referees and the readers. In keeping with this philosophy, we have developed a suite of policies and processes that aim to increase the rigour of peer review mechanisms, the robustness of published papers, and ultimately author satisfaction. As always, we welcome your feedback on these initiatives at cellbio@nature.com 